\title{
SHARP CAFFARELLI-KOHN-NIRENBERG INEQUALITIES ON STRATIFIED LIE GROUPS
}

\author{
Van Hoang Nguyen \\ Duy Tan University, Institute of Research and Development \\ Da Nang, Vietnam; vanhoang0610@yahoo.com
}

\begin{abstract}
In this paper, we prove a family of sharp Caffarelli-Kohn-Nirenberg inequalities on stratified Lie groups. Our result sharpens the inequalities obtained recently by Ruzhansky, Suragan and Yessirkegenov [22], and extend the classical Caffarelli-Kohn-Nirenberg inequalities to a new class of exponents (negative or smaller than 1) which we believe to be new in literature. Finally, we generalize our result to the more general setting of homogeneous groups with any homogeneous quasi-norm.
\end{abstract}

\section{Introduction}

Let us recall the classical Caffarelli-Kohn-Nirenberg (shortly, CKN) inequalities [2]: let $n \geq 1$ and let $p, q, r, \alpha, \beta, \gamma, \delta$ and $\sigma$ be real number such that

$$
p, q \geq 1, \quad r>0, \quad a \in[0,1]
$$

and

$$
\frac{1}{p}+\frac{\alpha}{n}>0, \quad \frac{1}{q}+\frac{\beta}{n}>0, \quad \frac{1}{r}+\frac{\gamma}{n}>0,
$$

where

$$
\gamma=\delta \sigma+(1-\delta) \beta
$$

Then there exists a positive constant $C$ such that the following inequality holds for any function $f \in C_{0}^{\infty}\left(\mathbf{R}^{n}\right)$

$$
\left(\int_{\mathbf{R}^{n}}|f|^{r}|x|^{r \gamma} d x\right)^{\frac{1}{r}} \leq C\left(\int_{\mathbf{R}^{n}}|f|^{p}|x|^{\alpha p} d x\right)^{\frac{\delta}{p}}\left(\int_{\mathbf{R}^{n}}|f|^{q}|x|^{\beta q} d x\right)^{\frac{1-\delta}{q}}
$$

if and only if the following conditions hold

$$
\frac{1}{r}+\frac{\gamma}{n}=\delta\left(\frac{1}{p}+\frac{\alpha-1}{n}\right)+(1-\delta)\left(\frac{1}{q}+\frac{\beta}{n}\right),
$$

(this is dimensional balance)

$$
\alpha-\sigma \geq 0 \quad \text { if } \quad \delta>0
$$

and

$$
\alpha-\sigma \leq 1 \quad \text { if } \quad \delta>0 \quad \text { and } \quad \frac{1}{r}+\frac{\gamma}{n}=\frac{1}{p}+\frac{\alpha-1}{n}
$$

https://doi.org/10.5186/aasfm.2018.4363

2010 Mathematics Subject Classification: Primary 26D10, 43A85, 22E30, 43A80.

Key words: Caffarelli-Kohn-Nirenberg inequalities, stratified Lie groups, sharp constants, optimal functions. 
The CKN inequalities (1.4) are a very useful tool in theorey of partial differential equations. Since its appearance in [2], such inequalities of this kind have been intensively studied in many setting. For examples, the CKN inequalities was generalized to the stratified Lie groups, the homogeneous groups, the metric measure spaces, to Riemannian manifolds with negative curvature and to derivatives of fractional order, etc. We refer the readers to $[5,7,8,6,13,15,16,17,18,20,21,24,23,25]$ for dicussions on this subject.

It is an interesting and non-trivial problem to look for the sharp constant in the CKN inequalities (1.4). In this direction, Xia proved a subfamily of (1.4) with an explicit constant (see [25])

$$
\left(\int_{\mathbf{R}^{n}} \frac{|f|^{r}}{|x|^{r \gamma}} d x\right)^{\frac{1}{r}} \leq \frac{r}{n-\gamma r}\left(\int_{\mathbf{R}^{n}} \frac{|\nabla f|^{p}}{|x|^{\alpha p}} d x\right)^{\frac{1}{p}}\left(\int_{\mathbf{R}^{n}} \frac{|f|^{\frac{p(r-1)}{p-1}}}{|x|^{\beta}} d x\right)^{\frac{p-1}{p}}
$$

for any function $f \in C_{0}^{\infty}\left(\mathbf{R}^{n}\right)$ where $r>p>1$ and $\alpha, \beta, \gamma$ satisfy

$$
\frac{1}{p}-\frac{\alpha}{n}>0, \quad 1-\frac{\beta}{n}>0, \quad \frac{1}{r}-\frac{\gamma}{n}>0,
$$

and

$$
\gamma=\frac{\alpha+1}{r}+\frac{p-1}{p r} \beta .
$$

The constant $r /(n-\gamma r)$ is sharp in (1.8) if

$$
n-\beta<\left(1+\alpha-\frac{\beta}{p}\right) \frac{p(r-1)}{p-1} .
$$

In this case, a family of optimal fuctions is given by

$$
f(x)=c\left(\lambda+|x|^{1+\alpha-\frac{\beta}{p}}\right)^{\frac{1-p}{r-p}}, \quad c \in \mathbf{R}, \lambda>0 .
$$

We refer the reader to $[4,14]$ in which the authors computed explicitly the sharp constant in the other cases of Caffarelli-Kohn-Nirenberg inequality.

The CKN inequalities on stratified Lie groups (or homogeneous Carnot groups) were recently established by Ruzhansky and Suragan [20]. Let $\mathbf{G}$ be a stratified Lie group with $N$ being the dimension of the first stratum and let $|\cdot|$ be the Euclidean norm on $\mathbf{R}^{N}$ (see Section 2 for more details on the stratified Lie groups). We denote by $x^{\prime}$ the variable from the first stratum of $G$. Then, Ruzhansky ans Suragan proved the following inequality

$$
\frac{|N-\gamma p|}{p}\left\|\frac{f}{\left|x^{\prime}\right|^{\gamma}}\right\|_{L^{p}(\mathbf{G})}^{p} \leq\left\|\frac{\nabla_{H} f}{\left|x^{\prime}\right|^{\alpha}}\right\|_{L^{p}(\mathbf{G})}\left\|\frac{f}{\left|x^{\prime}\right|^{\frac{\beta}{p}}}\right\|_{L^{p}(\mathbf{G})}^{p},
$$

for any $f \in C_{0}^{\infty}\left(\mathbf{G} \backslash\left\{x^{\prime}=0\right\}\right)$ where $1<p<\infty, \gamma p=1+\alpha+\frac{p-1}{p} \beta$, and $\nabla_{H}$ denotes the horizontal gradient on $\mathbf{G}$. Furthermore, if $N \neq \gamma p$ then the constant $\frac{|N-\gamma p|}{p}$ is sharp. The case $\beta=p \gamma$, the inequality (1.12) covers the sharp weighted Hardy inequality for horizontal gradient on stratified Lie groups (see [20] for the discussions).

The inequality (1.12) recently was extended to a larger range of parameters as follows (see [22]): let $p, q>1, r>0$ with $p+q \geq r$ and $\delta \in[0,1] \cap\left[\frac{r-q}{r}, \frac{p}{r}\right]$ and 
$a, b, c \in \mathbf{R}$ such that $\frac{\delta r}{p}+\frac{(1-\delta) r}{q}=1$ and $c=\delta(a-1)+b(1-\delta)$. Then the following inequality holds for any function $f \in C_{0}^{\infty}\left(\mathbf{G} \backslash\left\{x^{\prime}=0\right\}\right)$

$$
\left\|\left|x^{\prime}\right|^{c} f\right\|_{L^{r}(\mathbf{G})} \leq\left|\frac{p}{N+p(a-1)}\right|^{\delta}\left\|\left|x^{\prime}\right|^{a} f\right\|_{L^{p}(\mathbf{G})}^{\delta}\left\|\left|x^{\prime}\right|^{b} f\right\|_{L^{q}(\mathbf{G})}^{1-\delta} .
$$

The constant $|p /(N+p(a-1))|^{\delta}$ is sharp for $p=q$ with $a-b=1$ or $p \neq q$ with $p(1-a)+q b \neq 0$ or $\delta=0,1$. The proof of (1.13) given in [22] is the applications of the Hölder inequality and the horizontal weighted $L_{p}$-Hardy inequality on $\mathbf{G}$. The applications of these two inequalities lose the sharpness of (1.13) in some cases. It is worthy to mention here that the inequalities (1.12) and (1.13) was generalized to the homogeneous spaces with any homogeneous quasi-norm in [18, 24, 23].

Our aim of this paper is two folds. Firstly, we look for the sharp form for a subfamily of the CKN inequality (1.13). Secondly, we extend the inequality (1.13) to a new range of parameters in which $q$ does not need to be greater than 1 , even we prove an inequality for negative $q$. Our main result reads as follows

Theorem 1.1. Let $\mathbf{G}$ be a stratified Lie groups with $N$ being the dimension of the first stratum, and let $|\cdot|$ denote the Euclidean norm on $\mathbf{R}^{N}$. Suppose that $p>1$, $r>0$ and $\alpha, \beta, \gamma \in \mathbf{R}$ such that

$$
\gamma=\frac{1+\alpha}{r}+\frac{p-1}{p r} \beta .
$$

Then the following inequalities hold for any $f \in C_{0}^{\infty}\left(\mathbf{G} \backslash\left\{x^{\prime}=0\right\}\right)$

(i) If $r>1$ then we have

$$
\int_{\mathbf{G}} \frac{|f|^{r}}{\left|x^{\prime}\right|^{\gamma r}} d x \leq \frac{r}{|N-\gamma r|}\left(\int_{\mathbf{G}} \frac{\left|\nabla_{H} f\right|^{p}}{\left|x^{\prime}\right|^{\alpha p}} d x\right)^{\frac{1}{p}}\left(\int_{\mathbf{G}} \frac{|f|^{\frac{p(r-1)}{p-1}}}{\left|x^{\prime}\right|^{\beta}} d x\right)^{\frac{p-1}{p}},
$$

where $d x$ denotes the Haar measure on $\mathbf{G}$.

(ii) If $0<r<1$ then we have

$$
\int_{\mathbf{G}} \frac{|f|^{r}}{\left|x^{\prime}\right|^{\gamma r}} d x \leq \frac{r}{|N-\gamma r|}\left(\int_{\mathbf{G}} \frac{\left|\nabla_{H} f\right|^{p}}{\left|x^{\prime}\right|^{\alpha p}} d x\right)^{\frac{1}{p}}\left(\int_{\operatorname{supp} f} \frac{|f|^{\frac{p(r-1)}{p-1}}}{\left|x^{\prime}\right|^{\beta}} d x\right)^{\frac{p-1}{p}},
$$

where supp $f$ denotes the support of $f$.

Furthermore, the inequalities (1.15) and (1.16) are sharp.

Let us comment something about Theorem 1.1. The difference between (1.15) and (1.16) is the last integral in (1.16) is taken on the support of $f$ (not all $\mathbf{G}$ ). The fact here is that when $r \in(0,1)$ then $p(r-1) /(p-1)<0$ so we must take the integral on the support of $f$ to make the inequality (1.16) being sense. The inequality (1.16) gives an example of CKN inequality with negative exponent on stratified Lie groups which we believe to be new. If $r>p>1,(1.15)$ gives a generalization of the result of Xia (the inequality (1.8)) to the stratified Lie groups. The sharpness of (1.15) sharpens the CKN inequality due to Ruzhansky, Suragan and Yessirkegenov (1.13). The case $1<r<\frac{2 p-1}{p}$ of part (i) also seems to be new since in this case $q=p(r-1) /(p-1)<(0,1)$, thus $(1.15)$ extends $(1.13)$ to $q \in(0,1)$. In the abelian case $\mathbf{G}=\mathbf{R}^{n}$, Theorem 1.1 recovers the inequalities obtained recently by the author [16]. Moreover, in [17], the author proved the CKN inequalities of type (1.15) and (1.16) on Cartan-Hadamard manifolds (complete, simply connected Riemannian manifolds with negative sectional curvature), but with an extra condition 
(1.9). Several rigidity results for complete Riemannian manifolds supporting the sharp CKN inequalities of type (1.15) and (1.16) was also discussed in that paper.

Finally, we emphasize here that our results in Theorem 1.1 with the same best constant can be easily extended to more general setting of the homogeneous groups with any homogeneous quasi-norm (see Section 3 below).

The rest of this paper is organized as follows. In Section 2, we recall briefly the necessary concepts in the theory of stratified Lie groups and fix notation. In Section 3, we prove the CKN inequalities (1.15) and (1.16) (i.e., prove Theorem 1.1) and discuss about their generalization to the homogeneous groups.

\section{Preliminaries}

We recall briefly, in this section, the basic concepts in the theory of stratified Lie groups. A Lie group $\mathbf{G}=\left(\mathbf{R}^{n}, \circ\right)$ is called a stratified group (or a homogeneous Carnot group) if the following conditions holds:

(i) For some natural numbers $N_{1}, N_{2}, \ldots, N_{r}$ (with $N=N_{1}$ ) such that $n=$ $N+N_{2}+\cdots+N_{r}$, the decomposition $\mathbf{R}^{n}=\mathbf{R}^{N} \times \mathbf{R}^{N_{2}} \times \cdots \times \mathbf{R}^{N_{r}}$ is valid, and for any $\lambda>0$, the dilation $\delta_{\lambda}: \mathbf{R}^{n} \rightarrow \mathbf{R}^{n}$ given by

$$
\delta_{\lambda}(x)=\delta_{\lambda}\left(x^{\prime}, x^{(2)}, \ldots, x^{(r)}\right):=\left(\lambda x^{\prime}, \lambda^{2} x^{(2)}, \ldots, \lambda^{r} x^{(r)}\right)
$$

is an automorphism of the group $\mathbf{G}$. Here $x^{\prime}=x^{(1)} \in \mathbf{R}^{N}$ and $x^{(k)} \in \mathbf{R}^{\left(N_{k}\right)}$ for $k=2, \ldots, r$.

(ii) Let $N$ be as in (i) and let $X_{1}, \ldots, X_{N}$ be the left invariant vector fields on $\mathbf{G}$ such that $X_{k}(0)=\left.\frac{\partial}{\partial x_{k}}\right|_{0}$ for $k=1,2, \ldots, N$. Then

$$
\operatorname{rank}\left(\operatorname{Lie}\left\{X_{1}, X_{2}, \ldots, X_{N}\right\}\right)=n,
$$

for any $x \in \mathbf{R}^{n}$, that is, the iterated commutators of $X_{1}, X_{2}, \ldots, X_{N}$ span the Lie algebra of $\mathbf{G}$.

If these two conditions (i) and (ii) hold, we then say that the triple $\mathbf{G}=\left(\mathbf{R}^{n}, \circ, \delta_{\lambda}\right)$ is a stratified group. Such groups have been thoroughly investigated by Folland [11]. We refer the reader to [1] for more details on stratified Lie groups and to [10] for more detailed discussions from the Lie algebra point of view. The class of stratified Lie groups includes the Euclidean space (the abelian groups), the Heisenberg group as the main example, as well as more general H-type groups and other groups.

The number $r$ in the definition of the stratified Lie group above is called the step of $\mathbf{G}$. The left invariant vector fields $X_{1}, \ldots, X_{N}$ are called the (Jacobian) generators of $\mathbf{G}$ and the number

$$
Q=\sum_{k=1}^{r} k N_{k}, \quad N_{1}=N
$$

is called the homogeneous dimension of $\mathbf{G}$. The second order differential operator

$$
\mathcal{L}=\sum_{k=1}^{N} X_{k}^{2},
$$

is called the (canonical) sub-Laplacian on G. This is a left invariant homogeneous hypoelliptic differential operator and it is known that $\mathcal{L}$ is elliptic if and only if the step of $\mathbf{G}$ is equal to 1 . Let us recall that the standard Lebesgue measure $d x$ on $\mathbf{R}^{n}$ is the Haar measure on $\mathbf{G}$ (see [10, Proposition 1.6.6]). The left invariant vector field 
$X_{j}$ has an explicit form and satisfies the divergence theorem (see, e.g., [19] for more details). More precisely, we can write explicitly

$$
X_{k}=\frac{\partial}{\partial x_{k}^{\prime}}+\sum_{l=2}^{r} \sum_{m=1}^{N_{l}} a_{k, m}^{(l)}\left(x^{\prime}, \ldots, x^{(l-1)}\right) \frac{\partial}{\partial x_{m}^{(l)}},
$$

see also [10, Section 3.1.5] for a general presentation. Let us denote the horizontal gradient on $\mathbf{G}$ by

$$
\nabla_{H}=\left(X_{1}, \ldots, X_{N}\right)
$$

and the horizontal divergence by

$$
\operatorname{div}_{H} v=\nabla_{H} \cdot v
$$

Then the sub-Laplacian $\mathcal{L}$ has the form

$$
\mathcal{L} f=\operatorname{div}_{H}\left(\nabla_{H} f\right) .
$$

More general, for any $1<p<\infty$, the horizontal $p$-sub-Laplacian is defined by

$$
\mathcal{L}_{p} f=\operatorname{div}_{H}\left(\left|\nabla_{H} f\right|^{p-2} \nabla_{H} f\right),
$$

here for $x^{\prime}=\left(x_{1}^{\prime}, \ldots, x_{N}^{\prime}\right) \in \mathbf{R}^{N}$,

$$
\left|x^{\prime}\right|=\sqrt{x_{1}^{\prime 2}+\cdots+x_{N}^{\prime 2}}
$$

denotes the Euclidean norm on $\mathbf{R}^{N}$.

Using the explicit representation of the left invariant vector fields $X_{k}$ above, we can easily check that

$$
\left.\left.\left|\nabla_{H}\right| x^{\prime}\right|^{\gamma}|=\gamma| x^{\prime}\right|^{\gamma-1}
$$

and

$$
\operatorname{div}_{H}\left(\frac{x^{\prime}}{\left|x^{\prime}\right|^{\gamma}}\right)=\frac{N-\gamma}{\left|x^{\prime}\right|^{\gamma}}
$$

for any $\gamma \in \mathbf{R}$ and $\left|x^{\prime}\right| \neq 0$.

\section{Proof of Theorem 1.1}

In this section, we will adopt the notation from Section 2 to prove Theorem 1.1. The proof is quite simple by using integration by parts and Hölder inequality, but it gives us the sharp constant in a subclass of the CKN inequalities (1.13). Let us remark that in the abelian case $\mathbf{G}=\left(\mathbf{R}^{n},+\right)$ we then have $N=n, \nabla_{H}=\nabla=\left(\partial_{x_{1}}, \ldots, \partial_{x_{n}}\right)$. Theorem 1.1 hence implies the following subfamilies of CKN inequalities on $\mathbf{G}=\mathbf{R}^{n}$ : Let $p>1, r>0$ and $\alpha, \beta, \gamma \in \mathbf{R}$ such that the condition (1.14) holds. Then we have for any $f \in C_{0}^{\infty}\left(\mathbf{R}^{n} \backslash\{0\}\right)$

(i) If $r>1$, then

$$
\int_{\mathbf{R}^{n}} \frac{|f|^{r}}{|x|^{\gamma r}} d x \leq \frac{r}{|n-\gamma r|}\left(\int_{\mathbf{R}^{n}} \frac{|\nabla f|^{p}}{|x|^{\alpha p}} d x\right)^{\frac{1}{p}}\left(\int_{\mathbf{R}^{n}} \frac{|f|^{\frac{p(r-1)}{p-1}}}{|x|^{\beta}} d x\right)^{\frac{p-1}{p}} .
$$

(ii) If $r \in(0,1)$, then

$$
\int_{\mathbf{R}^{n}} \frac{|f|^{r}}{|x|^{\gamma r}} d x \leq \frac{r}{|n-\gamma r|}\left(\int_{\mathbf{R}^{n}} \frac{|\nabla f|^{p}}{|x|^{\alpha p}} d x\right)^{\frac{1}{p}}\left(\int_{\text {supp } f} \frac{|f|^{\frac{p(r-1)}{p-1}}}{|x|^{\beta}} d x\right)^{\frac{p-1}{p}} .
$$


Here $|x|=\sqrt{x_{1}^{2}+\cdots+x_{n}^{2}}$ denotes the Euclidean norm on $\mathbf{R}^{n}$ and $n \neq \gamma r$. The constant $r /|n-\gamma r|$ in the inequalities (3.1) and (3.2) is sharp. The inequalities (3.1) and (3.2) was recently proved by the author [16] under the extra conditions that

$$
\frac{1}{p}-\frac{\alpha}{n}>0, \quad \frac{1}{r}-\frac{\gamma}{n}>0, \quad 1-\frac{\beta}{n}>0 .
$$

While the inequality (3.1) was earlier proved by Xia [25] under the conditions $r>$ $p>1$ and (3.3). Furthermore, Xia proved that if the following extra assumption

$$
n-\beta<\left(1+\alpha-\frac{\beta}{p}\right) \frac{p(r-1)}{r-p}
$$

holds then a family of optimal functions of (3.1) is given by

$$
f(x)=c\left(\lambda+|x|^{1+\alpha-\frac{\beta}{p}}\right)^{\frac{p-1}{p-r}}, \quad c \in \mathbf{R}, \lambda>0 .
$$

For the case $0<r<p$ and $\alpha, \beta, \gamma$ satisfying (3.3), it was proved by the author [16] that the sharp constants in (3.1) and (3.1) are attained by

$$
f(x)=c\left(\lambda-|x|^{1+\alpha-\frac{\beta}{p}}\right)_{+}^{\frac{p-1}{p-r}}, \quad c \in \mathbf{R}, \lambda>0,
$$

if $1+\alpha-\frac{\beta}{p}>0$, where $a_{+}=\max \{a, 0\}$ denotes the positive part of a number $a$,

$$
f(x)=c\left(|x|^{1+\alpha-\frac{\beta}{p}}-\lambda\right)_{+}^{\frac{p-1}{p-r}}, \quad c \in \mathbf{R}, \lambda>0
$$

if $1+\alpha-\frac{\beta}{p}<0$ and $n-\beta+\left(1+\alpha-\frac{\beta}{p}\right) \frac{p(r-1)}{p-r}>0$, and

$$
f(x)=c(\lambda-\ln |x|)_{+}{ }^{\frac{p-1}{p-r}}, \quad c \in \mathbf{R}, \lambda \in \mathbf{R}
$$

if $1+\alpha-\frac{\beta}{p}=0$. The inequalities (3.1) and (3.2) provide several new inequalities of CKN type when $0<r<\frac{2 p-1}{p}, r \neq 1$ since in this case $q=\frac{p(r-1)}{p-1}<1$ (and maybe strict negative) which is different from the classical CKN inequalities (1.4) and from the inequality of Ruzhansky, Suragan and Yessirkegenov (1.13). We refer the reader to [17] for the generalizations of (3.1) and (3.2) to Cartan-Hadamard manifolds, and to a stronger versions where $\nabla f$ is replaced by radial derivative $\partial_{r} f$.

Proof of Theorem 1.1. For any function $f \in C_{0}^{\infty}\left(\mathbf{G} \backslash\left\{x^{\prime}=0\right\}\right)$, using (2.2) and integration by parts, we get for $N-\gamma r \neq 0$ that

$$
\begin{aligned}
(N-\gamma r) \int_{\mathbf{G}} \frac{|f|^{r}}{\left|x^{\prime}\right|^{\gamma r}} d x & =-\int_{\mathbf{G}}|f|^{r} \operatorname{div}_{H}\left(\frac{x^{\prime}}{\left|x^{\prime}\right|^{\gamma r}}\right) d x \\
& =-r \int_{\mathbf{G}} \frac{1}{\left|x^{\prime}\right|^{\gamma r-1}}|f|^{r-2} f \frac{x^{\prime}}{\left|x^{\prime}\right|} \cdot \nabla_{H} f d x,
\end{aligned}
$$

which easily implies

$$
\begin{aligned}
\int_{\mathbf{G}} \frac{|f|^{r}}{\left|x^{\prime}\right|^{\gamma r}} d x & \leq \frac{r}{|N-\gamma r|} \int_{\mathbf{G}} \frac{1}{\left|x^{\prime}\right|^{\gamma r-1}}|f|^{r-1}\left|\frac{x^{\prime}}{\left|x^{\prime}\right|} \cdot \nabla_{H} f\right| d x \\
& =\frac{r}{|N-\gamma r|} \int_{\mathbf{G}} \frac{|f|^{r-1}}{\left|x^{\prime}\right|^{\frac{p-1}{p} \beta}} \frac{1}{\left|x^{\prime}\right|^{\alpha}}\left|\frac{x^{\prime}}{\left|x^{\prime}\right|} \cdot \nabla_{H} f\right| d x
\end{aligned}
$$

here we used (1.14) which is equivalent to $\gamma r-1=\alpha+\frac{p-1}{p} \beta$. 
Suppose that $r>1$. Applying Hölder inequality to (3.5) yields

$$
\int_{\mathbf{G}} \frac{|f|^{r}}{\left|x^{\prime}\right|^{\gamma r}} d x \leq \frac{r}{|N-\gamma r|}\left(\int_{\mathbf{G}} \frac{\left|\frac{x^{\prime}}{\left|x^{\prime}\right|} \cdot \nabla_{H} f\right|^{p}}{\left|x^{\prime}\right|^{\alpha p}} d x\right)^{\frac{1}{p}}\left(\int_{\mathbf{G}} \frac{|f|^{\frac{p(r-1)}{p-1}}}{\left|x^{\prime}\right|^{\beta}} d x\right)^{\frac{p-1}{p}} .
$$

Since $\left|\frac{x^{\prime}}{\left|x^{\prime}\right|} \cdot \nabla_{H} f\right| \leq\left|\nabla_{H} f\right|$ then (3.6) implies our desired inequality (1.15). It should be mentioned here that in the abelian case $\mathbf{G}=\left(\mathbf{R}^{n},+\right)$, we have $\frac{x^{\prime}}{\left|x^{\prime}\right|} \cdot \nabla_{H} f=\partial_{r} f$ the radial derivative of $f$. Hence (3.6) implies (3.1). However, the inequality (3.6) in the Euclidean space was already proved in [16].

Suppose that $r \in(0,1)$. In this case, (3.4) is replaced by

$$
(N-\gamma r) \int_{\mathbf{G}} \frac{|f|^{r}}{\left|x^{\prime}\right|^{\gamma r}} d x=-r \int_{\operatorname{supp} f} \frac{1}{\left|x^{\prime}\right|^{\gamma r-1}}|f|^{r-2} f \frac{x^{\prime}}{\left|x^{\prime}\right|} \cdot \nabla_{H} f d x,
$$

which implies

$$
\begin{aligned}
\int_{\mathbf{G}} \frac{|f|^{r}}{\left|x^{\prime}\right|^{\gamma r}} d x & \leq \frac{r}{|N-\gamma r|} \int_{\operatorname{supp} f} \frac{1}{\left|x^{\prime}\right|^{\gamma r-1}}|f|^{r-1}\left|\frac{x^{\prime}}{\left|x^{\prime}\right|} \cdot \nabla_{H} f\right| d x \\
& =\frac{r}{|N-\gamma r|} \int_{\operatorname{supp} f} \frac{|f|^{r-1}}{\left|x^{\prime}\right|^{\frac{p-1}{p} \beta}} \frac{1}{\left|x^{\prime}\right|^{\alpha}}\left|\frac{x^{\prime}}{\left|x^{\prime}\right|} \cdot \nabla_{H} f\right| d x .
\end{aligned}
$$

Applying Hölder inequality to (3.7) and the fact $\left|\frac{x^{\prime}}{\left|x^{\prime}\right|} \cdot \nabla_{H} f\right| \leq\left|\nabla_{H} f\right|$, we obtain the desired inequality (1.16).

It remains to check the sharpness of (1.15) and (1.16). To do this, we concentrate to treat the case of equality in Hölder inequality applied in the proof of (1.15) and (1.16), i.e.,

$$
\frac{x^{\prime}}{\left|x^{\prime}\right|} \cdot \nabla_{H} f=-c|f|^{\frac{r-p}{p-1}} f\left|x^{\prime}\right|^{\alpha-\frac{\beta}{p}}
$$

for some $c>0$ with remark that when $r \in(0,1)$ the equality holds on the support of $f$. We concentrate to find $f$ to be the nonnegative function of $\left|x^{\prime}\right|$, i.e., $f(x)=h\left(\left|x^{\prime}\right|\right)$. Then the equation (3.8) becomes

$$
h^{\prime}(s)=-c h(s)^{\frac{r-1}{p-1}} s^{\alpha-\frac{\beta}{p}}, \quad s>0,
$$

for some $c>0$ with remark that when $r \in(0,1)$ the equality holds on $(0, R)$ with $R=\sup \left\{\left|x^{\prime}\right|: x^{\prime} \in \operatorname{supp} f\right\}$. The explict solutions of (3.9) are as follows depending on $p, r$ and $\alpha, \beta, \gamma$ :

(i) If $1<p<r$ and $1+\alpha-\frac{\beta}{p} \neq 0$, then

$$
h(s)=\left(\lambda+c \frac{r-p}{p-1} \frac{s^{1+\alpha-\frac{\beta}{p}}}{1+\alpha-\frac{\beta}{p}}\right)_{+}^{\frac{p-1}{p-r}}
$$

for some $c, \lambda>0$.

(ii) If $1<p<r$ and $1+\alpha-\frac{\beta}{p}=0$, then

$$
h(s)=\left(\lambda+c \frac{r-p}{p-1} \ln s\right)_{+}^{\frac{p-1}{p-r}}
$$

for some $c>0$ and $\lambda \in \mathbf{R}$. 
(iii) If $0<r<p$ and $1+\alpha-\frac{\beta}{p} \neq 0$, then

$$
h(s)=\left(\lambda-c \frac{p-r}{p-1} \frac{s^{1+\alpha-\frac{\beta}{p}}}{1+\alpha-\frac{\beta}{p}}\right)_{+}^{\frac{p-1}{p-r}},
$$

for some $c, \lambda>0$.

(iv) If $0<r<p$ and $1+\alpha-\frac{\beta}{p}=0$, then

$$
h(s)=\left(\lambda-c \frac{p-r}{p-1} \ln s\right)_{+}^{\frac{p-1}{p-r}}
$$

for some $c>0$ and $\lambda \in \mathbf{R}$.

(v) If $p=r$ and $1+\alpha-\frac{\beta}{p} \neq 0$, then

$$
h(s)=\lambda e^{-\frac{c}{1+\alpha-\frac{\beta}{p}} s^{1+\alpha-\frac{\beta}{p}}}
$$

for some $c, \lambda>0$.

(vi) If $p=r$ and $1+\alpha-\frac{\beta}{p}=0$, then

$$
h(s)=\lambda s^{-c}
$$

for some $c, \lambda>0$.

From these expressions of $h$, we obtain the explicit function $f$ in (3.8). By approximating this function $f$ on $\mathbf{R}^{N}$ and the constant function on $\mathbf{R}^{N_{2}} \times \cdots \times \mathbf{R}^{N_{r}}$ by compactly supported function, we obtain the sharpness of (1.15) and (1.16) (see [9] for a special case $p=q=r$ in $\mathbf{R}^{n}$ ).

We conclude this section by remark that Theorem 1.1 and its proof can be easily extended to the more general setting of homogeneous groups (which contains the stratified Lie groups as the main examples). The analysis on homogeneous groups was developed by Folland and Stein [12]. For more recent developments, we refer the reader to [10]. Let us briefly recall some basic notions of homogeneous groups. A homogeneous group $\mathbf{G}$ is a simply connected Lie group equipped with a dilation structure defined as follows. Let $\mathfrak{g}$ denote the Lie algebra of $\mathbf{G}$. We consider a family of dilations on $\mathfrak{g}$ as follows

$$
D_{\lambda}=\operatorname{Exp}(A \ln \lambda)=\sum_{k=0}^{\infty} \frac{1}{k !}(A \ln \lambda)^{k}, \quad \lambda>0,
$$

where $A$ is a diagonalizable positive linear operator on $\mathfrak{g}$. Each dilation $D_{\lambda}$ is a morphism of $\mathfrak{g}$, i.e., a linear map from $\mathfrak{g}$ to its self with compatible with Lie bracket on $\mathfrak{g}$, that is,

$$
\left[D_{\lambda} X, D_{\lambda} Y\right]=D_{\lambda}[X, Y], \quad \lambda>0, X, Y \in \mathfrak{g} .
$$

Since the exponential map $\operatorname{Exp}_{\mathbf{G}}: \mathfrak{g} \rightarrow \mathbf{G}$ is a global diffeomorphism, it induces a dilation structure on $\mathbf{G}$ (still denoted by $D_{\lambda}(x)$ or $\lambda x$ )

$$
D_{\lambda}(x)=\lambda x:=\operatorname{Exp}_{\mathbf{G}}\left(D_{\lambda}\left(\operatorname{Exp}_{\mathbf{G}}^{-1} x\right)\right), \quad x \in \mathbf{G} .
$$

The homogeneous dimension of $\mathbf{G}$ is $Q=\operatorname{Tr} A$. Let $X_{1}, \ldots, X_{n}$ denote a fix basis of $\mathfrak{g}$ such that $A X_{k}=\nu_{k} X_{k}$, i.e., $A$ is diagonalized in this basis of the form $A=$ $\operatorname{diag}\left(\nu_{1}, \ldots, \nu_{n}\right)$. Obviously, $Q=\nu_{1}+\cdots+\nu_{n}$. For each $x \in \mathbf{G}$, we denote by $\left(e_{1}(x), \ldots, e_{n}(x)\right)$ the coordinate of $\operatorname{Exp}_{\mathbf{G}}^{-1} x$ in this basis, i.e.,

$$
\operatorname{Exp}_{\mathbf{G}}^{-1} x=e_{1}(x) X_{1}+\cdots+e_{n}(x) X_{n} .
$$


Thus, the dilation $D_{\lambda}$ on $\mathbf{G}$ has the form

$$
D_{\lambda} x=\lambda x=\operatorname{Exp}_{\mathbf{G}}\left(\lambda^{\nu_{1}} e_{1}(x) X_{1}+\cdots+\lambda^{\nu_{n}} e_{n}(x) X_{n}\right),
$$

or equivalently, $e_{i}(\lambda x)=\lambda^{\nu_{i}} e_{i}(x)$. A direct computation shows that

$$
\frac{d}{d r} f(r x)=\left(\sum_{k=1}^{n} \nu_{k} r^{\nu_{k}-1} X_{k} f\right)(r x) \text {. }
$$

Define

$$
\mathcal{R}=\sum_{k=1}^{n} \nu_{k} \frac{e_{k}(x)}{|x|} X_{k}
$$

we then have

$$
\frac{d}{d r} f(r x)=\mathcal{R} f(r x)
$$

That is $\mathcal{R}$ is homogeneous of order -1 . It plays the role of the radial derivative on G. Evidently, $\mathcal{R}=\partial_{r}$ in the abelian case $\mathbf{G}=\mathbf{R}^{n}$.

Let $|\cdot|: \mathbf{G} \rightarrow(0, \infty)$ be a homogeneous quasi-norm on $\mathbf{G}$, i.e., $\left|x^{-1}\right|=|x|$, $|\lambda x|=\lambda|x|$ for $\lambda \geq 0$, and $|x|=0$ if and only if $x=0$. It is well-known that every homogeneous groups admit a homogeneous quasi-norm. Let $\mathfrak{G}$ denote the quasi-unit sphere with respect to $|\cdot|$, i.e.,

$$
\mathfrak{G}=\{x \in \mathbf{G}:|x|=1\} .
$$

There exists unique positive Borel measure $\sigma$ on $\mathfrak{G}$ such that

$$
\int_{\mathbf{G}} f d x=\int_{0}^{\infty} \int_{\mathfrak{G}} f(r y) r^{Q-1} d \sigma(y) d r
$$

where $d x$ denotes the Haar measure on $\mathbf{G}$ which is homogeneous of order $Q$ (see $[12,10]$ for the proof of this statement).

With these notions and notation, we can generalize Theorem 1.1 to the homogeneous group $\mathbf{G}$ with homogeneous quasi-norm $|\cdot|$ as follows.

Theorem 3.1. Let $\mathbf{G}$ be a homogeneous group of homogeneous dimension $Q$ and let $|\cdot|$ be any homogeneous quasi-norm on $\mathbf{G}$. Let $p>1, r>0$ and $\alpha, \beta, \gamma$ such that $\gamma=\frac{\alpha+1}{r}+\frac{p-1}{p r} \beta$. Then the following inequalities holds for any functions $f \in C_{0}^{\infty}(\mathbf{G} \backslash\{0\})$

(i) If $r>1$ then we have

$$
\int_{\mathbf{G}} \frac{|f|^{r}}{|x|^{\gamma r}} d x \leq \frac{r}{|Q-\gamma r|}\left(\int_{\mathbf{G}} \frac{|\mathcal{R} f|^{p}}{|x|^{\alpha p}} d x\right)^{\frac{1}{p}}\left(\int_{\mathbf{G}} \frac{|f|^{\frac{p(r-1)}{p-1}}}{|x|^{\beta}} d x\right)^{\frac{p-1}{p}} .
$$

(ii) If $0<r<1$ then we have

$$
\int_{\mathbf{G}} \frac{|f|^{r}}{|x|^{\gamma r}} d x \leq \frac{r}{|Q-\gamma r|}\left(\int_{\mathbf{G}} \frac{|\mathcal{R} f|^{p}}{|x|^{\alpha p}} d x\right)^{\frac{1}{p}}\left(\int_{\operatorname{supp} f} \frac{|f|^{\frac{p(r-1)}{p-1}}}{|x|^{\beta}} d x\right)^{\frac{p-1}{p}} .
$$

Furthermore, the constant $r /|Q-\gamma r|$ is sharp in (3.11) and (3.12) for any homogeneous quasi-norm.

Theorem 3.1 was proved in [18] for $p=r$. The inequality of type (3.11) was proved in $[24,23]$ for a larger class of parameters $p, r$ and $\alpha, \beta, \gamma$ but without sharp constant. We believe that the inequalities (3.11) with $1<r<\frac{2 p-1}{p}$ and (3.12) are 
new in the setting of homogeneous groups. And they maybe suggest us a more general class of CKN inequality with new type of exponents (smaller than 1 or negative).

The proof of Theorem 3.1 is similar with the one of Theorem 1.1. Instead of using divergence theorem and (2.2), we use the formula (3.10) and integration by parts as follows.

$$
\begin{aligned}
\int_{\mathbf{G}} \frac{|f|^{r}}{|x|^{\gamma r}} d x & =\int_{0}^{\infty} \int_{\mathfrak{G}}|f(s y)|^{r} s^{Q-r \gamma-1} d s d \sigma(y) \\
& =-\frac{r}{Q-r \gamma} \int_{0}^{\infty} \int_{\mathfrak{G}}|f(s y)|^{r-2} f(s y) \mathcal{R} f(s y) s^{Q-r \gamma} d s d \sigma \\
& =-\frac{r}{Q-r \gamma} \int_{\mathbf{G}} \frac{|f|^{r-2} f}{|x|^{\beta \frac{p-1}{p}}} \frac{\mathcal{R} f}{|x|^{\alpha}} d x .
\end{aligned}
$$

We now can apply Hölder inequality to obtain our desired inequalities with remark that if $r \in(0,1)$ we take the integral in the support of $f$.

The sharpness of $r /|Q-r \gamma|$ when $Q-r \gamma \neq 0$ is verified as in the proof of Theorem 1.1. Furthermore, we see that this sharp constant is attained in several cases as follows:

(i) $1<p<r$ and $n-\beta<\left(1+\alpha-\frac{\beta}{p}\right) \frac{p(r-1)}{r-p}$. A family of optimal functions is given by

$$
f(x)=c\left(\lambda+|x|^{1+\alpha-\frac{\beta}{p}}\right)^{\frac{p-1}{p-r}}, \quad c \in \mathbf{R}, \lambda>0 .
$$

(ii) $0<r<p, r \neq 1$ and $1+\alpha-\frac{\beta}{p}>0$. A family of optimal functions is given by

$$
f(x)=c\left(\lambda-|x|^{1+\alpha-\frac{\beta}{p}}\right)_{+}^{\frac{p-1}{p-r}}, \quad c \in \mathbf{R}, \lambda>0 .
$$

(iii) $0<r<p, r \neq 1$ and $1+\alpha-\frac{\beta}{p}=0$. A family of optimal functions is given by

$$
f(x)=c(\lambda-\ln |x|)_{+}^{\frac{p-1}{p-r}}, \quad c \in \mathbf{R}, \lambda \in \mathbf{R} .
$$

(iv) $0<r<p, r \neq 1,1+\alpha-\frac{\beta}{p}<0$ and $n-\beta+\left(1+\alpha-\frac{\beta}{p}\right) \frac{p(r-1)}{p-r}>0$. A family of optimal functions is given by

$$
f(x)=c\left(|x|^{1+\alpha-\frac{\beta}{p}}-\lambda\right)_{+}^{\frac{p-1}{p-r}}, \quad c \in \mathbf{R}, \lambda>0 .
$$

\section{References}

[1] Bonfiglioli, A., E. Lanconelli, and F. Uguzzoni: Stratified Lie groups and potential theory for their sub-Laplacians. - Springer, Berlin, 2007.

[2] Caffarelli, L., R. Kohn, and L. Nirenberg: First order interpolation inequalities with weights. - Compositio Math. 53, 1984, 259-275.

[3] Do CARmo, M.P., and C. XiA: Complete manifolds with non-negative Ricci curvature and the Caffarelli-Kohn-Nirenberg inequalities. - Compositio Math. 140, 2004, 818-826.

[4] Chanillo, A. L., S. Chanillo, and A. Maalaoui: Constant norm in cases of the CaffarelliKohn-Nirenberg inequality. - Pacific J. Math. 292:2, 2018, 293-303.

[5] Cordero-Erausquin, D., B. Nazaret, and C. Villani: A mass-transportation approach to sharp Sobolev and Gagliardo-Nirenberg inequalities. - Adv. Math. 182, 2004, 307-332.

[6] Costa, D. G.: Some new and short proofs for a class of Caffarelli-Kohn-Nirenberg type inequalities. - J. Math. Anal. Appl. 337, 2008, 311-317.

[7] Del Pino, M., and J. Dolbeault: Best constants for Gagliardo-Nirenberg inequalities and applications to nonlinear diffusions. - J. Math. Pures Appl. 81, 2002, 847-875. 
[8] Del Pino, M., and J. Dolbeault: The optimal Euclidean $L_{p}$-Sobolev logarithmic inequality. - J. Funct. Anal. 197, 2003, 151-161.

[9] Di, Y., L. JiAng, S. Shen, and Y. Jin: A note on a class of Hardy-Rellich type inequalities. - J. Inequal. Appl. 84, 2013, 1-6.

[10] Fisher, V., and M. Ruzhansky: Quantization on nilpotent Lie groups. - Progr. Math. 314, Birkhäuser, 2016.

[11] Folland, G. B.: Subelliptic estimates and function spaces on nilpotent Lie groups. - Ark. Mat. 13, 1975, 161-207.

[12] Folland, G. B., and E. M. Stein: Hardy spaces on homogeneous groups. - Math. Notes 28, Princeton Univ. Press, Princeton, N.J.; Univ. of Tokyo Press, Tokyo, 1982.

[13] HaN, Y.: Weighted Caffarelli-Kohn-Nirenberg type inequality on the Heisenberg group. Indian J. Pure Appl. Math. 46, 2015, 147-161.

[14] Lam, N., and G. Lu: Sharp constants and optimizers for a class of Caffarelli-Kohn-Nirenberg inequalities. - Adv. Nonlinear Stud. 17:3, 2017, 457-480.

[15] Nguyen, H. M., and M. Squassina: Fractional Caffarelli-Kohn-Nirenberg inequalities. - J. Funct. Anal. 274, 2018, 2661-2672.

[16] Nguyen, V. H.: New sharp Hardy and Rellich type inequalities on Cartan-Hadamard manifolds and their improvements. - arXiv:1708.0936.

[17] Nguyen, V. H.: Sharp Caffarelli-Kohn-Nirenberg inequalities on Riemannian manifolds: the influence of curvature. - arXiv:1709.06120.

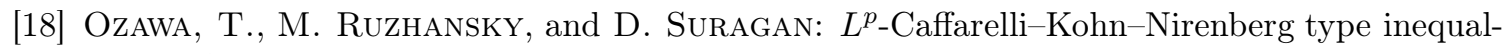
ities on homogeneous groups. - arXiv:1605.02520.

[19] Ruzhansky, M., and D. Suragan: Layer potentials, Kac's problem, and refined Hardy inequality on homogeneous Carnot groups. - Adv. Math. 308, 2017, 483-528.

[20] Ruzhansky, M., and D. Suragan: On horizontal Hardy, Rellich, Caffarelli-Kohn-Nirenberg and $p$-sub-Laplacian inequalities on stratified groups. - J. Differential Equations 262, 2017, 1799-1821.

[21] Ruzhansky, M., and D. Suragan: Anisotropic $L^{2}$-weighted Hardy and $L^{2}$-Caffarelli-KohnNirenberg inequalities. - Commun. Comtemp. Math. 19, 2017, 1750014 (12 pages).

[22] Ruzhansky, M., D. Suragan, and N. Yessirkegenov: Caffarelli-Kohn-Nirenberg and Sobolev type inequalities on stratified Lie groups. - Nonlinear Differ. Equ. Appl. 24:56, 2017.

[23] Ruzhansky, M., D. Suragan, and N. Yessirkegenov: Extended Caffarelli-KohnNirenberg inequalities and superweights for $L^{p}$-weighted Hardy inequalities. - C. R. Acad. Sci. Paris 355, 2017, 694-698.

[24] Ruzhansky, M., D. Suragan, and N. Yessirkegenov: Extended Caffarelli-KohnNirenberg inequalities, and remainders, stability, and superweights for $L^{p}$-weighted Hardy inequalities. - Trans. Amer. Math. Soc. Ser. B 5, 2018, 32-62.

[25] XIA, C.: The Caffarelli-Kohn-Nirenberg inequalities on complete manifolds. - Math. Res. Lett. $14,2007,875-885$.

Received 2 March $2018 \bullet$ Accepted 1 June 2018 